University of Nebraska - Lincoln

DigitalCommons@University of Nebraska - Lincoln

$5-4-2006$

\title{
Functional replacement of the ketosynthase domain of FUM1 for the biosynthesis of fumonisins, a group of fungal reduced polyketides
}

\author{
Xiangcheng Zhu \\ University of Nebraska - Lincoln \\ Fengan $\mathrm{Yu}$ \\ University of Nebraska - Lincoln, fyu2@unl.edu \\ R. S. Bojja \\ University of Nebraska - Lincoln \\ K. Zaleta-Rivera \\ University of Nebraska - Lincoln \\ Liangcheng Du \\ University of Nebraska-Lincoln, Idu3@unl.edu
}

Follow this and additional works at: https://digitalcommons.unl.edu/chemistrydu

Part of the Chemistry Commons

Zhu, Xiangcheng; Yu, Fengan; Bojja, R. S.; Zaleta-Rivera, K.; and Du, Liangcheng, "Functional replacement of the ketosynthase domain of FUM1 for the biosynthesis of fumonisins, a group of fungal reduced polyketides" (2006). Liangcheng Du Publications. 5.

https://digitalcommons.unl.edu/chemistrydu/5

This Article is brought to you for free and open access by the Published Research - Department of Chemistry at DigitalCommons@University of Nebraska - Lincoln. It has been accepted for inclusion in Liangcheng Du Publications by an authorized administrator of DigitalCommons@University of Nebraska - Lincoln. 
Published in Journal of Industrial Microbiology \& Biotechnology 33 (2006), pp. 859-868. doi 10.1007/s10295-006-0137-9 Journal of Industrial Microbiology \& Biotechnology is the official journal published by Springer Verlag for the Society for Industrial Microbiology. Copyright (C) 2006 Society for Industrial

Microbiology. Used by permission. http://www.springerlink.com/content/100967/

\title{
Functional replacement of the ketosynthase domain of FUM1 for the biosynthesis of fumonisins, a group of fungal reduced polyketides
}

\author{
X. Zhu, F. Yu, R. S. Bojja, K. Zaleta-Rivera, and L. Du* \\ Department of Chemistry, University of Nebraska-Lincoln, Lincoln, NE 68588-0304 \\ *Corresponding author. Email: $\underline{1 \mathrm{du} @ \text { unlserve.unl.edu }}$
}

\begin{abstract}
The genetic manipulation of the biosynthesis of fungal reduced polyketides has been challenging due to the lack of knowledge on the biosynthetic mechanism, the difficulties in the detection of the acyclic, non-aromatic metabolites, and the complexity in genetically manipulating filamentous fungi. Fumonisins are a group of economically important mycotoxins that contaminate maize-based food and feed products worldwide. Fumonisins contain a linear dimethylated $\mathrm{C}_{18}$ chain that is synthesized by Fumlp, which is a single module polyketide synthase (PKS). Using a genetic system that allows the specific manipulation of PKS domains in filamentous fungus Fusarium verticillioides, we replaced the KS domain of fumonisin FUM1 with the KS domain of T-toxin PKS1 from Cochliobolus heterostrophus. Although PKS1 synthesizes different polyketides, the $F$. verticillioides strain carrying the chimeric PKS produced fumonisins. This represents the first successful domain swapping in PKSs for fungal reduced polyketides and suggests that KS domain alone may not be sufficient to control the product's structure. To further test if the whole fumonisin PKS could be functionally replaced by a PKS that has a similar domain architecture, we replaced entire FUM1 with PKS1. This strain did not produce any fumonisin or new metabolites, suggesting that the intrinsic interactions between the intact PKS and downstream enzymes in the biosynthetic pathway may play a role in the control of fungal reduced polyketides.
\end{abstract}

Keywords: Biosynthesis, Fumonisin, Polyketide synthase, Fusarium verticillioides

\section{Introduction}

Fungal polyketide synthases (PKSs) are modular enzymes typically consisting of a single set of domains [25]. These domains function iteratively during the assembly of the polyketide chain, which is in contrast to bacterial modular PKSs (Type I). Typical bacterial modular PKSs function non-iteratively, and the number, order, and domain-composition of the modules dictate the length and reduction level of the polyketide product [25]. Although data from heterologous expression and in vitro assays of a number of fungal PKSs, for example 6-methylsalicylic acid synthase $[3,13,22]$ and squalestatin tetraketide synthase [8], have shown that each of the single module fungal PKS contains the necessary information for synthesizing a distinct polyketide product, it is not clear how the single module enzymes determine the chain-length of products. The understanding of the biosynthetic mechanism is critical to the production of new industrially important fungal polyketide metabolites, such as the cholesterol-lowering drug lovastatin, via genetic manipulations of the biosynthetic genes.

Information regarding the biosynthetic mechanism for fungal polyketides has emerged in the recent years. Most of the studies were conducted with PKSs synthesizing fungal aromatic polyketides. The architecture of PKSs for aromatic polyketides, such as naphthopyrone synthase WA in Aspergillus nidulans [11], is very different from that for reduced polyketides, such as fumonisin Fum1p [21] and lovastatin LNKS [15]. Fungal PKSs for aromatic polyketides are characterized by having a thioesterase-like domain, Claisen cyclase (CYC) domain, at the $C$-terminus [11]. This domain has recently been shown to contribute to the chain length determination of fungal aromatic polyketides [31]. On the other hand, fungal PKSs for reduced polyketides do not have a CYC domain and therefore must require a discrete mechanism to release the polyketide with a specific chain-length from PKS.

We have been studying a group of fungal reduced polyketides, fumonisins, to understand the biosynthetic mech- 
anism for fungal reduced polyketides $[5,10,29,33,34]$. Fumonisins are mycotoxins produced by Fusarium verticillioides, which is a widespread fungal contaminant of corn and maize-based food and feeds [17]. Fumonisins have a linear carbon backbone that is of polyketide origin (Fig. 1) [21]. Isotope feeding experiments have established that carbons 3-20 of fumonisins are derived from acetate and carbons 1-2 and the amino group on $\mathrm{C}-2$ are from alanine $[4,6]$. The two methyl groups at $\mathrm{C}-12$ and $\mathrm{C}-16$ are derived from methionine [19]. The hydroxyl oxygen on C-5, C-10, C-14, and C-15 of fumonisin $\mathrm{B}_{1}\left(\mathrm{FB}_{1}\right)$ are derived from molecular oxygen, whereas the hydroxyl oxygen on $\mathrm{C}-3$ is from the carbonyl group of acetate [7]. A cluster of 15 genes (FUM) for fumonisin biosynthesis has been cloned from $F$. verticillioides [20, 21, 24], among which is a PKS gene (FUMI). The disruption of FUM1 led to the elimination of fumonisin production [21], showing that this gene is responsible for the biosynthesis of fumonisins.

FUM1 was predicted to code for a PKS with 7-domain (KSAT-DH-MT-ER-KR-ACP) (Fig. 1) [21]. This organization is almost identical to several other PKSs for the biosynthesis of reduced polyketides, such as LDKS and LNKS for lovastatin in A. terreus [15] and PKS1 for T-toxins in Cochliobolus heterostrophus [32], although the polyketide chain-length of fumonisins, lovastatin, and T-toxins is substantially different from each other. The objectives of this study were to test whether a heterologous PKS is functionally interchangeable with FUM1 in $F$. verticillioides and whether such a swapping would result in polyketide products with an altered chain-length. In light of the recent information regarding the chain-length determination by the heterodimeric ketosynthase-chain length factor (KS-CLF, also known as $\mathrm{KS}_{\alpha}-\mathrm{KS}_{\beta}$ ) in the biosynthesis of bacterial aromatic polyketides $[14,28]$, the outcomes from the fungal KS domain swapping experiments may shed light on the mechanism for the biosynthesis of fungal reduced polyketides.

\section{Materials and methods}

\section{Materials and strains}

Fusarium verticillioides wild-type strain A0149 was provided by Dr. David Gilchrist (University of California, Davis). YPD medium and V-8 juice agar were respectively used to produce mycelia and conidia [21], and cracked maize kernels (CMK) for fumonisin production $[7,21]$.

Preparation and analysis of nucleic acids

Escherichia coli DH5- $\alpha$ strain was used as the host for general DNA preparation, and the pGEM-zf vector series from Promega (Madison, WI, USA) for cloning and DNA sequencing. Plasmid preparation and DNA extraction were carried out using Qiagen kits (Valencia, CA, USA), and all other manipulations were carried out according to standard methods [23]. Genomic DNA of $F$. verticillioides was prepared as described previously [9]. In experiments using PCR to quickly screen putative mutants, genomic DNA was mini-prepared from the mutants by using UltraClean ${ }^{\mathrm{TM}}$ Microbial DNA Isolation Kit (Mo Bio,

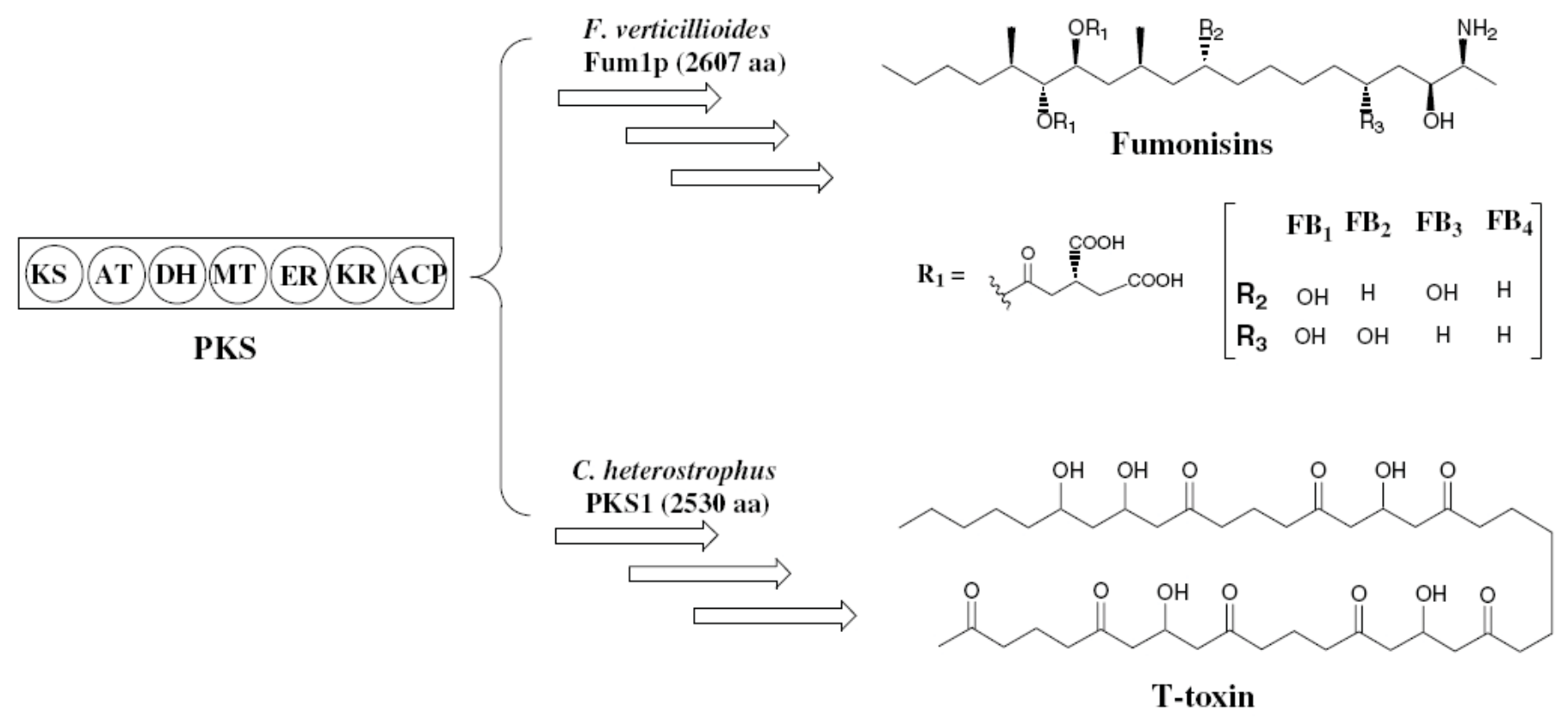

Figure 1 The domain organization of fumonisin Fum1p and T-toxin PKS1 and the chemical structure of the metabolites (only the best characterized $\mathrm{C}_{41}$ analog of T-toxins shown). Abbreviations: $\beta$-ketoacyl synthase (KS), acyl- transferase $(A T)$, dehydratase $(D H)$, methyltransferase $(M T)$, enoylreductase $(E R), \beta$-ketoacyl reductase $(K R)$, and acyl carrier protein $(A C P)$ 
CA, USA). Southern hybridization was performed by using DIG DNA labeling and Detection Kit (Roche, Indianapolis, IN, USA) according to the manufacturer's manual. Approximately $10 \mu \mathrm{g}$ of genomic DNA was used in the hybridizations, and Immobilon $^{\mathrm{TM}}-\mathrm{NY}^{+}$Transfer Membrane (Millipore, Bedford, MA, USA) was used to transfer the DNA. The probe-KS $(1.3 \mathrm{~kb}$, Fig. 3d, f) was amplified by PCR using primers tKS-F/tKS-R (Table 1) with $C$. heterostrophus clone pF5P1 [32] as template. The probe $\mathrm{t} 1$ (703 bp) and $\mathrm{t} 2(681 \mathrm{bp})$ in Fig. 5 were prepared using primers PKS1-1-F/PKS1-1-R and PKS1-2-F/PKS1-2-R (Table 1), respectively, with $\mathrm{pF} 5 \mathrm{P} 1$ as template. The probe $\mathrm{f1}$ (800 bp) and f3 (737 bp) in Fig. 5 were amplified by PCR using primers FUM1-1-F/FUM1-1-R and FUM1-3-F/FUM1-3-R (Table 1), respectively, with Cos6B [21] as template. RT-PCR was carried out using a kit from Invitrogen (Carlsbad, CA, USA). Total RNA was isolated from the cultures using the TRIzol method provided in the manufacturer's manual. The RNA was treated with Amp DNase I (Invitrogen) for $15 \mathrm{~min}$ at $25^{\circ} \mathrm{C}$ and used in the first-strand cDNA synthesis using SuperScript ${ }^{\mathrm{TM}}$ First-strand Synthesis System (Invitrogen). A reaction did not contain the reverse transcriptase was included as a control. A pair of primers, P1/P2 (Table 1), was used in the RT-PCR.

Construction of plasmid pZXC-lw92-KS for KS domain replacement

To construct the plasmid for replacing the KS domain of FUM1, the XhoI/SacI fragment of pUCH2-8 containing the hygromycin resistance gene $H y g B$ (also known as $h p h$ or $h p t$ ) [1] was cloned into the same sites of pSP72 to form pUCHSP. A $5.7 \mathrm{~kb} \mathrm{NsiI}$ fragment, which contains the 5'-noncoding region $(1.1 \mathrm{~kb})$ and the 5 -coding region of FUM1 gene, was obtained from Cos6B [21] by NsiI digestion. After purifica- tion from gel, this fragment was further digested with SmaI and $B g l \mathrm{II}$ to yield a $4.3 \mathrm{~kb}$ fragment, which was cloned into SmaI/BglII sites of pUCH-SP to yield pUCH-fKS. A 1,280-bp fragment coding for the KS domain of T-toxin PKS1 was amplified by PCR from $C$. heterostrophus clone pF5P1 [32] using primers tKS-F and tKS-R (Table 1). The fidelity of this PCR fragment was confirmed by DNA sequencing and it was then digested with $S p h \mathrm{I} / K p n \mathrm{I}$. pUCH-fKS was also digested with $S p h \mathrm{I} / K p n \mathrm{I}$, and the larger fragment was isolated and ligated with the $S p h \mathrm{I} / K p n \mathrm{I}$ digested PCR fragment to produce $\mathrm{pZXC}$ 1w92-KS (Fig. 3a). This construct contains the KS domain of T-toxin PKS1 flanked by the upstream region (461 bp) and the downstream region $(2,645 \mathrm{bp})$ of the KS domain of FUM1.

Construction of plasmid pUCH-LFUM1-PKS1-RFUM1 for intact PKS replacement

To construct the PKS gene replacement vector, pUCHLFUM1-PKS1-RFUM1, a 1,096 bp DNA fragment (LFUM1) was amplified by PCR using primers of L-FUM1-F/L-FUM1$\mathrm{R}$ (Table 1), and $F$. verticillioides clone Cos6B as template. This fragment is the upstream flanking region of FUM1 gene. It was digested with NotI and SpeI and cloned into the same sites of pBlueScript SK(+) to yield pBSK-LFUM1. A 547 bp fragment (PKS1-N) was amplified by PCR using primers of PKS1-N-F/PKS1-N-R and C. heterostrophus clone pF5P1 as template. This fragment was digested with SpeI and EcoRI and cloned into pBSK-LFUM1 to produce pBSK-LFUM1-PKS1N. A 274 bp fragment (PKS1-C) was also amplified by PCR using primers of PKS1-C-F/PKS1-C-R and C. heterostrophus clone $\mathrm{pF} 5 \mathrm{P} 1$ as template. It was digested with HindIII and ApaI and cloned into the same sites of pBSK-LFUM1-PKS1N to yield pBSK-LFUM1-PKS1N-C. pBSK-LFUM1-PKS1N-C

\begin{tabular}{ll}
\hline Primers & Sequences \\
\hline tKS-F & 5'-TTGCTAAGCATGCTTTCTGAGGGCAGA-3' \\
tKS-R & 5'-ACACTCTAAAGTTGTAGGTACC-3' \\
P1 & 5'-CTTGCGGCCGGTTCTAGC-3' \\
P2 & 5'-GTTGACGCTGACCCTAAG-3' \\
P3 & 5'-CAGTGTTATTGGAGACTC-3' \\
P4 & 5'-AACCCAGAATTCGTACATTGG-3' \\
L-FUM1-F & 5'-GCATGCATGCGGCCGCCCCAAGCAATTTTGCAGA- \\
L-FUM1-R & 5'-GACTAGTCGTTGATGATATCAGTAGCG-3' \\
PKS1-N-F & 5'-CACTAGTATGACAGTTCGCGATTCTAAAAC-3' \\
PKS1-N-R & 5'-AACCCAGAATTCGTACATTGG-3' \\
PKS1-C-F & 5'-CAATCCTCAAGCTTTGATAGT-3' \\
PKS1-C-R & 5'-TGAAAAGGGCCCTTAACCCCTATTATTTGT-3' \\
R-FUM1-F & 5'-CCATAGGGGCCCACCTAGACACTGAGCTGG-3' \\
R-FUM1-R & 5'-GGACCCTCGCATATGCAGCAGGTCACGGAAGCA-3 \\
FUM1-1-F & 5'-TCCAAGGTTTAGAGGTGAGTCTG-3' \\
FUM1-1-R & 5'-CGAGAAGAAGATTAGGTATCGGG-3' \\
FUM1-3-F & 5'-CAACTCCCTTCCTGAGATAAACA-3' \\
FUM1-3-R & 5'-CTGTAGACCTTGCCTTCCAAAA-3' \\
PKS1-1-F & 5'-ATTTCCAGTATCCAGCCTCTACC-3' \\
PKS1-1-R & 5'-TCGTTTCCCATCTCTCTCATC-3' \\
PKS1-2-F & 5'-GTGCTACTGCTTTGTGGATGTC-3' \\
PKS1-2-R & 5'-CAGGAAGTGAAGTGTCAGGATG-3' \\
\hline
\end{tabular}


was then digested with NotI and ApaI, and the LFUM1-PKS1N-C fragment was cloned into pANT841 to yield pANTLFUM1-PKS1N-C. To prepare the downstream flanking region (RFUM1) of FUM1 gene, a fragment of 1,345 bp was amplified by PCR using primers of R-FUM1-F/R-FUM1-R and $\operatorname{Cos} 6 \mathrm{~B}$ as template. This fragment was digested with ApaI and NdeI and cloned into the same sites of pANT-LFUM1PKS1N-C to produce pANT-LFUM1-PKS1NC-RFUM1. This construct contains the upstream (LFUM1) and downstream (RFUM1) flanking regions of $F$. verticillioides $F U M 1$ gene, as well as the $N$-terminus (PKS1N) and $C$-terminus (PKS1C) of C. heterostrophus $P K S 1$ gene. To prepare an intact $P K S 1$ gene in the construct, a 6,999 bp fragment was released from pF5P1 by EcoRI and HindIII digestion and cloned into the same sites of pANT-LFUM1-PKS1NC-RFUM1 to produce pANTLFUM1-PKS1-RFUM1. This clone contains intact PKS1 gene flanked by the upstream and downstream regions of FUM1 gene, which is a cassette ready for gene replacement by homologous recombination. To introduce an antibiotic selection marker $(H y g B)$ for fungal transformants, pANT-LFUM1PKS1-RFUM1 was digested with NdeI, followed by Klenow Fragment 3'-end fill-in. The DNA was further digested with NotI to release the whole gene replacement cassette. Finally, this cassette was cloned into NotI/SmaI sites of pUCH2-8 [1] to produce pUCH-LFUM1-PKS1-RFUM1 (Fig. 5a).

Transformation of $F$. verticillioides and screening for the mutants

The protocol used to isolate and transform protoplasts has been described previously [34]. The plasmid DNA $(5 \mu \mathrm{g})$ was diluted with STC buffer $(100 \mu$ f final) and mixed with protoplasts $(100 \mu \mathrm{l})$, and transformation was mediated with PEG 8000 buffer (30\% PEG 8000; 10 mM Tris-HCl, pH 8.0; $50 \mathrm{mM} \mathrm{CaCl}_{2}$ ). Hygromycin-resistant colonies were selected on YPD plates containing hygromycin B $(150 \mu \mathrm{g} / \mathrm{ml}$, Calbiochem, La Jolla, CA, USA). The screening of mutants involves two stages, which was also described in the previous work [34]. The first stage is to select for gene disruption mutants resulted from a single crossover of the homologous regions, whereas the second stage is to select for domain-replaced mutants by screening for the loss of hygromycin B resistance.

\section{Analysis of metabolites}

The single-conidia derived wild type strain or mutants were inoculated in CMK medium $(25 \mathrm{~g})$ and allowed to grow at $25^{\circ} \mathrm{C}$ in dark for 4 weeks as described previously $[5,10]$. The CMK cultures were extracted with water/acetonitrile $(1: 1, \mathrm{v} /$ v). The extracts were filtered and subjected to HPLC-ELSD or LC-ESMS analysis [5]. A HPLC system (ProStar, Model 210, Varian, Walnut Creek, CA, USA) coupled to an evaporative light-scattering detector (ELSD2000, Alltech, Deerfield, IL, USA) was used to analyze the extracts. The column was Alltima C18LL $(5 \mu, 250 \times 4.6 \mathrm{~mm}$ i.d., Alltech, Deerfield, IL, USA), and the experimental conditions were the same as described previously [5]. A RFC 18 Vydac MassSpec column $\left(5 \mu, 250 \times 1 \mathrm{~mm}\right.$ i.d., Vydac $\left.{ }^{\mathrm{TM}}\right)$ was used in the LC-ESMS analysis. All positive electrospray spectra were acquired using a Q-Tof Mass spectrometer (Micromass, Manchester, UK).

\section{Results}

\section{Generation of the KS-replaced mutants}

Two restriction enzyme sites, $S p h \mathrm{I}$ and $K p n \mathrm{I}$, are conveniently located at the upstream and downstream boundary, respectively, of the FUM1 KS domain (fKS). The boundaries were defined by Pileup comparison (GCG Program) of sequences from bacterial type I PKSs and fungal PKSs and following the domain definition in previous studies $[2,27]$. The sequence of fKS shows an identity/similarity of $41.8 \% / 58.2 \%$ to the KS domain (tKS) of T-toxin PKS1 (Fig. 2) [32]. A $K p n I$ site is also found at the downstream boundary of tKS domain. This site is located at the same position as the KpnI site of FUMI in the Pileup comparison. These features facilitated the replacement of fKS by tKS. The approach for the replacement is homologous recombination. The pSP72based construct (pZXC-1w92-KS) contains the hygromycin resistance gene $H y g B$ from pUCH2-8 [1]. The upstream and downstream homologous regions of the construct were directly cloned from cosmid Cos6B [21], which contains the FUM gene cluster, and the tKS domain was directly inserted into the $S p h \mathrm{I} / K p n \mathrm{I}$ sites of the construct. The fidelity of the replaced domain and the fusion regions between FUM1 and tKS were confirmed by DNA sequencing. Thus, a region of 362 amino acid residues (from number 56 to 417) of Fum1p (AAD43562) was replaced with a region of 366 residues (from number 38 to 403) of PKS1 (AAB08104) (Fig. 2).

Using the two-stage screening strategy, we obtained approximately three to seven hygromycin-resistant colonies using $5 \mu \mathrm{g}$ of plasmid DNA mixed with $5 \times 10^{7}$ protoplasts. PCR using a pair of primers binding to the $H y g B$ gene confirmed that all colonies contained the $\operatorname{Hyg} B$ gene (data not shown). One clone (L5) was confirmed by Southern blot to result from a homologous recombination, and the rest resulted from random insertions (data not shown). This single crossover event occurred at the fKS downstream homologous region (2,645 bp in length on plasmid ZXC-1w92-KS, Fig. 3a, b). For the second stage screening, L5 strain was cultured for five generations without hygromycin in the medium to encourage the second crossover in the homologous regions, either at the upstream to KS to produce domain-replaced mutants or at the downstream to KS to revert to the wild-type. Approximately 600 colonies were picked and individually inoculated on grid-lined plates, one copy on YPD and one on YPD/Hygromycin plates. Among the 600 colonies, four (K1, $2,3,4)$ lost the ability to grow on YPD/Hygromycin plates.

We first used PCR to identify the KS-replaced mutants. Two pairs of primers (P1/P2 and P3/P4, Table 1 and Fig. 3a) were used for PCR. For each pair of primers, one primer 
Figure 2 Sequence comparison of the KS domain of Fum1p and T-toxin PKS1. The identical residues are highlighted, and the conserved catalytic site cysteine residue is underlined and in a bold face letter.

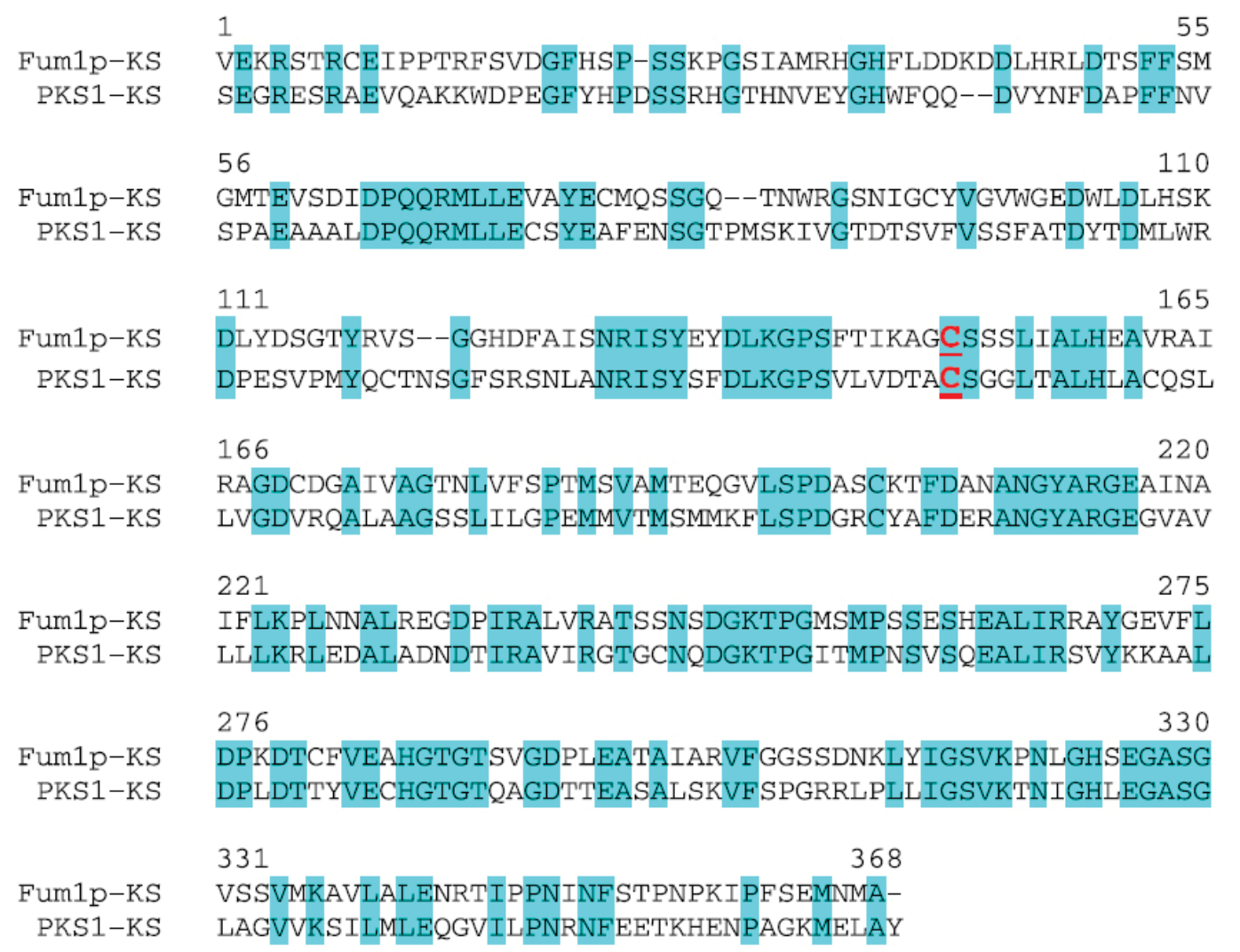
.

10
GMTEVSDIDPQQRMLLEVAYECMQSSGQ--TNWRGSNIGCYVGVWGEDWLDLHSK

RAGDCDGAIVAGTNLVFSPTMSVAMTEQGVLSPDASCKTFDANANGYARGEAINA

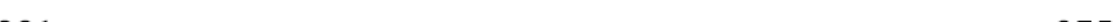

IFLKPLNNALREGDPIRALVRATSSNSDGKTPGMSMPSSESHEALIRRAYGEVFL LLLKRLEDALADNDTIRAVIRGTGCNQDGKTPGITMPNSVSQEALIRSVYKKAAL

\section{6}

330

DPKDTCFVEAHGTGTSVGDPLEATAIARVFGGSSDNKLYIGSVKPNLGHSEGASG

$331 \quad 368$

LAGVVKSILMLEQGVILPNRNFEETKHENPAGKMELAY binds to tKS and the other to the downstream or upstream of fKS on FUM1. Thus, the revertant (wild-type) may be distinguished from the domain replaced mutants. As expected, PCR amplified a 742 bp fragment from plasmid pZXC-1w92$\mathrm{KS}$ (positive control) using primers $\mathrm{P} 1 / \mathrm{P} 2$, but not from the wild-type strain (negative control) (Fig. 3d). Among the putative mutants, $\mathrm{K} 1, \mathrm{~K} 2$, and $\mathrm{K} 3$ gave the expected band, whereas K4 did not give the band (Fig. 3d). The results suggest that $\mathrm{K} 1, \mathrm{~K} 2$, and $\mathrm{K} 3$ contained the chimeric PKS gene and $\mathrm{K} 4$ did not contain this gene. K2 and K4 were also subjected to a second test by PCR using primers P3/P4. The plasmid and K2 gave the expected $687 \mathrm{bp}$, whereas K4 and the wild-type did not give any product (Fig. 3e). The fidelity of the PCR fragments was confirmed by DNA sequencing. Southern hybridization was conducted to further confirm the identity of these mutants. The genomic DNA and plasmid DNA were digested with EcoRI and probed with Dig-labeled tKS. The plasmid pZXC-1w92-KS was included as a control to produce two signals at $1.6 \mathrm{~kb}$ and $954 \mathrm{bp}$ (Fig. 3f). The wild-type and K4 strain did not give any signal, confirming that $\mathrm{K} 4$ is a revertant. $\mathrm{K} 2$ gave two signals $(7.8,1.6 \mathrm{~kb})$ expected for a KS-replaced mutant (Fig. 3f).

tKS domain was predicated to contain three small introns (74, 57, $41 \mathrm{bp})$ [32]. To test if the chimeric PKS could be transcripted in $F$. verticillioides, a RT-PCR was performed using primer $\mathrm{P} 1$ and $\mathrm{P} 2$. The primer site for $\mathrm{P} 1$ is located upstream to intron-2 (57 bp), and that for P2 is downstream to intron3 (41 bp). If the PKS is correctly transcripted in the host, the expected RT-PCR product should be $98 \mathrm{bp}$ shorter than the size of PCR product amplified from genomic DNA. Indeed, the RT-PCR data showed that a band of approximately $644 \mathrm{bp}$ was amplified from RNA prepared from K2 mutant, which is approximately $98 \mathrm{bp}$ smaller than the $742 \mathrm{bp}$ product amplified from the control (Data not shown).

Metabolites in the KS-replaced mutants

The metabolites produced in the mutants as well as in the wild-type of $F$. verticillioides were analyzed by high performance liquid chromatography-evaporative light-scattering detection (HPLC-ELSD) and liquid chromatography-electrospray ionization mass spectrometry (LC-ESMS). The extracts from the wild-type gave one major peak at the retention time of $15.3 \mathrm{~min}$ (Fig. $4 \mathrm{a}$ ) and a $[\mathrm{M}+\mathrm{H}]^{+}$of $722.8 \mathrm{~m} / \mathrm{z}$, which is identical to standard $\mathrm{FB}_{1}$. The yield of $\mathrm{FB}_{1}$ as estimated from HPLC-ELSD was approximately $0.7-1.5 \mathrm{mg} / \mathrm{g}$ CMK medium. Three minor peaks appeared at 16.1, 16.8, and $18.2 \mathrm{~min}$, which were coincident with standard $\mathrm{FB}_{3}, \mathrm{FB}_{2}$, and $\mathrm{FB}_{4}$, respectively (Fig. $4 \mathrm{a}$ ). They gave a $[\mathrm{M}+\mathrm{H}]^{+}$of 706.5 , 706.3 , and $690.4 \mathrm{~m} / \mathrm{z}$, respectively, which are the molecular mass expected for $\mathrm{FB}_{3}, \mathrm{FB}_{2}$, and $\mathrm{FB}_{4}$. The $\mathrm{L} 5$ strain that resulted from a single crossover did not produce any fumonisins (data not shown). This result is expected because FUM1 gene in this strain was disrupted by the plasmid insertion (Fig. 3b). $\mathrm{K} 4$ produced $\mathrm{FB}_{1}, \mathrm{FB}_{2}$, and $\mathrm{FB}_{3}$ with a level similar to the wild-type (data not shown). This is consistent with the South- 
Figure 3 Screening and confirmation of KS domain replaced mutants. a Homologous recombination between FUM1 on chromosome of $F$. verticillioides and the sequences on plasmid pZXC-1w92-KS. The asterisk indicates the position of the start codon of FUM1. The positions for PCR primer binding sites in the screening of the mutants are indicated by small arrows. Abbreviations: Bg: BglII; E: EcoRI; K: KpnI; Sm: SmaI; Sh: SphI; $H y g B$, hygromycin B resistant gene. b Gene disruption mutants resulted from a single crossover at the downstream homologous region. The position of the tKS probe used for Southern hybridization is indicated by a bar. c KS domain replaced mutant resulted from a second crossover at the homologous regions. $\mathbf{d}$ and $\mathbf{e}$ Confirmation of the mutants by PCR using primers P1/P2 (d) and P3/P4 (e), respectively. Lane-1, size markers; lane-2, wildtype; lane-3, K2; lane-4, K4; lane-5, plasmid. $\mathbf{f}$ Confirmation of mutants by Southern hybridization. The DNA was digested with $E c o$ RI and probed with a DIG-labeled KS domain of PKS1 as shown in $\mathbf{c}$. The sizes of hybridizing signals are indicated with arrows. Lane-1, size markers; lane-2, wildtype; lane-3, K2; lane-4, K4; lane-5, plasmid
A

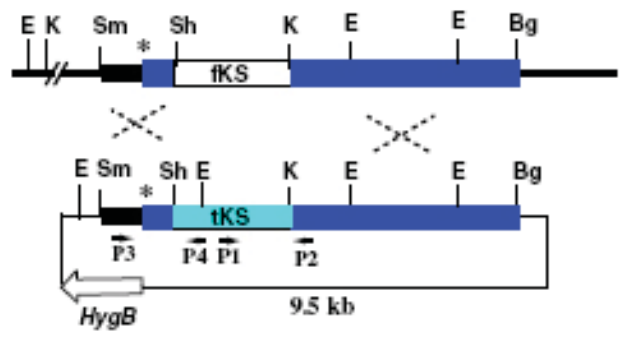

$F$. verticillioides chromosome (FUMI)

pZXC-1w92-KS

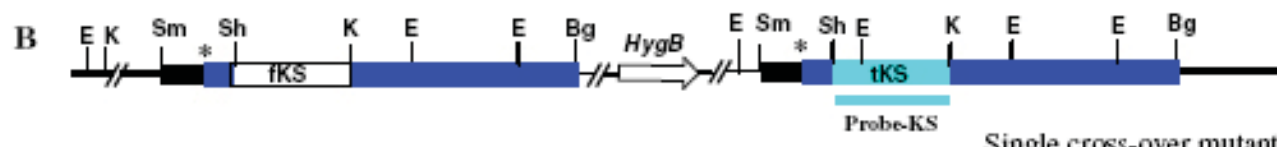

C
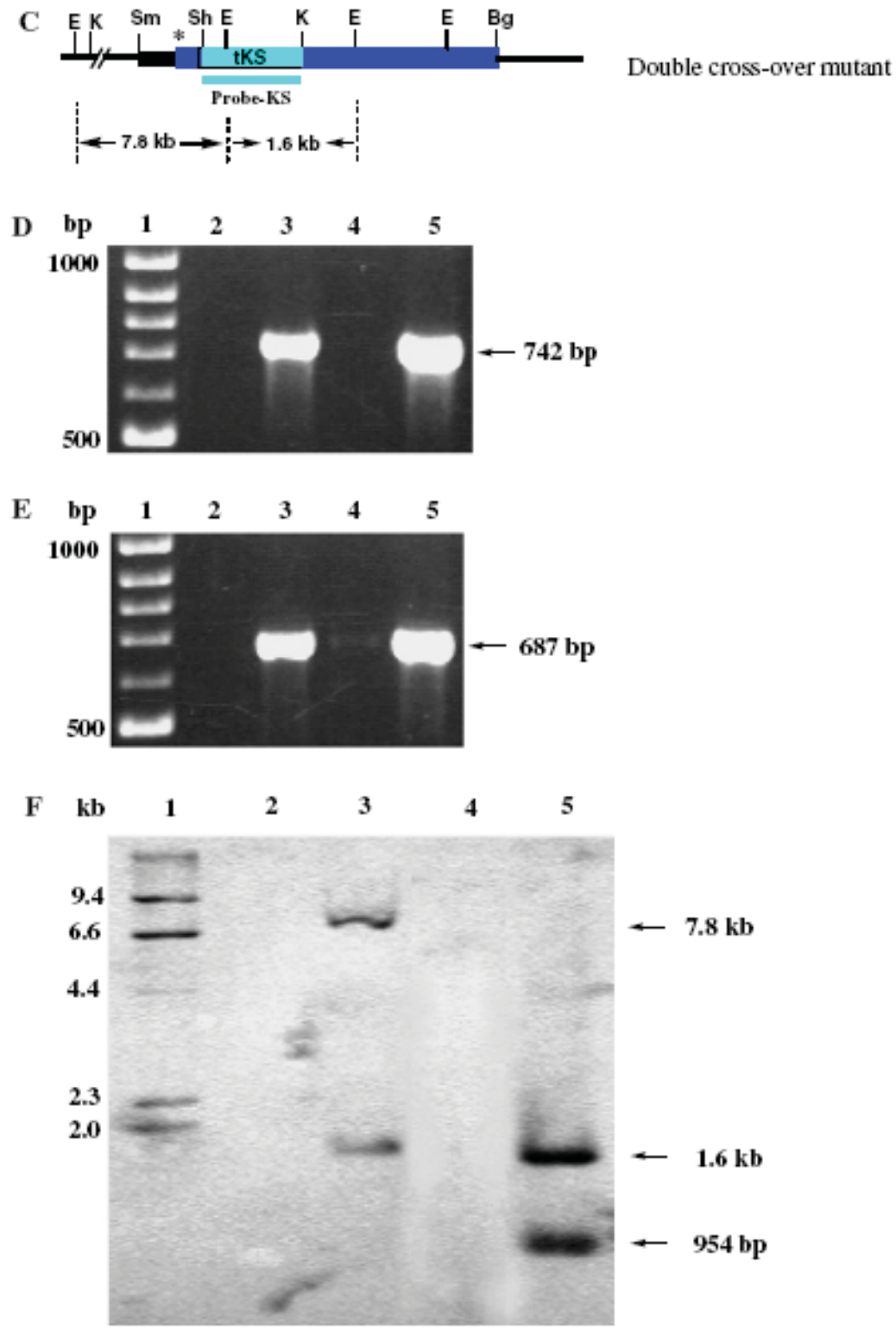

ern hybridization and PCR results, which showed that K4 is a revertant. The KS-replaced mutant $\mathrm{K} 2$ produced a pattern of fumonisins similar to the wild-type (Fig. 4b). The peaks at 15.1, 15.7, and 16.2 min gave a $[\mathrm{M}+\mathrm{H}]^{+}$of $722.9,706.4$, and
$706.3 \mathrm{~m} / \mathrm{z}$, respectively (Fig. 4c, d). The results show that tKS domain is functional in the chimeric FUM1 and that the fumonisin pathway could tolerate an individual domain swapping in the PKS. 

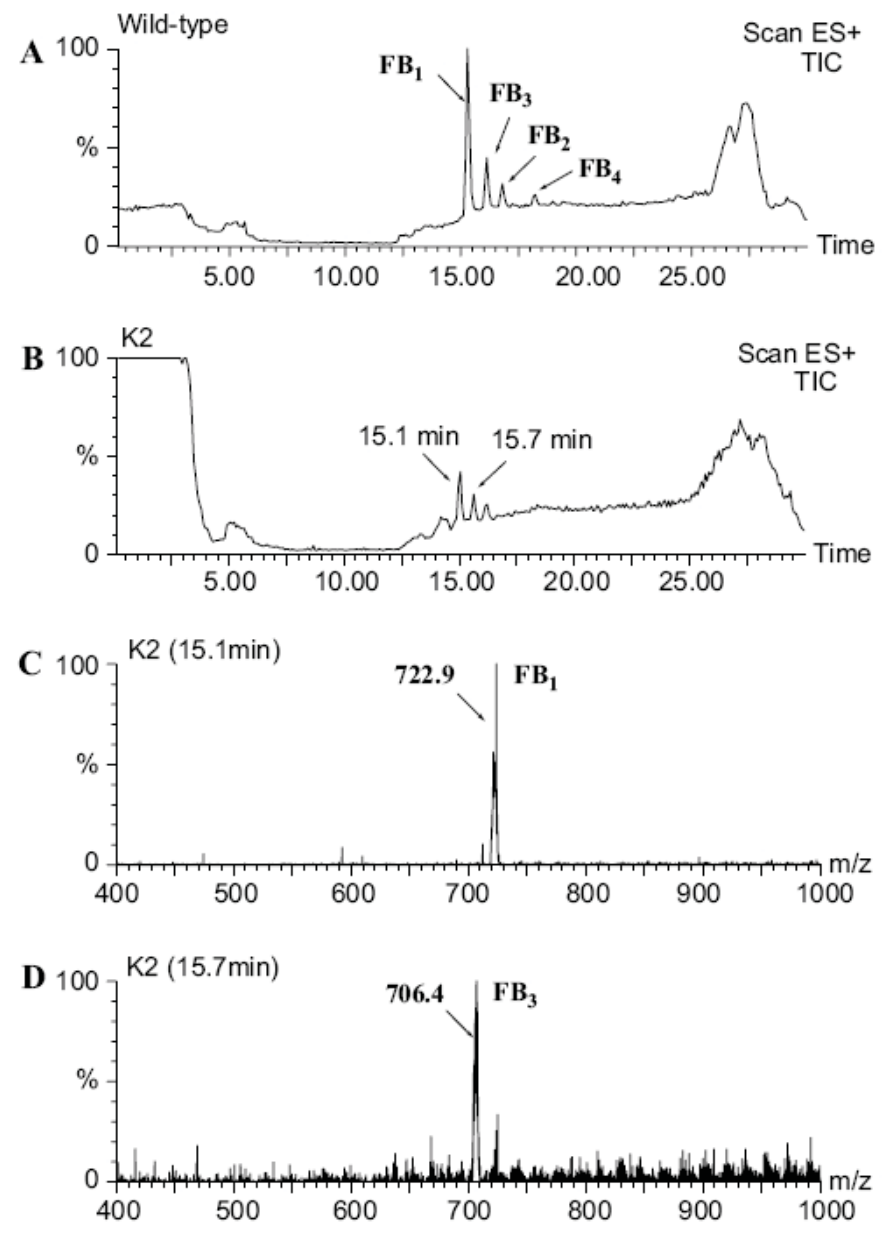

Figure 4 LC-ESMS analysis of metabolites isolated from the wild-type strain and the KS-replaced mutant. a LC of the extracts from the wild-type strain, with the peaks of fumonisins indicated. b LC of the extracts from K2 mutant, with the retention times of the major peaks indicated. $\mathbf{c}$ and $\mathbf{d}$ are the MS of the peak at 15.1 and 15.7 min, respectively, on the LC of K2 in b

Generation of the intact $F U M 1$-replaced mutants and analysis of metabolites

To further test if the entire fumonisin PKS could be functionally replaced by a PKS that has similar domain architecture, we replace the intact fumonisin FUM1 in F. verticillioides with T-toxin PKS1 from C. heterostrophus [32]. A plasmid, pUCH-LFUM1-PKS1-RFUM1, was constructed to contain the intact $P K S 1$ gene flanked by the upstream $(1,096 \mathrm{bp})$ and downstream $(1,345 \mathrm{bp})$ non-coding regions of FUM1 (Fig. 5a). Using the two-stage screening strategy, we first obtained 58 hygromycin-resistant colonies. PCR analyses of these putative transformants indicated that five resulted from homologous recombination, with four from the upstream homologous region and one at the downstream (Fig. 5b, c). The single cross-over mutants were then used to select for double cross-over mutants. We obtained 43 colonies lost the hygromycin resistance. PCR analyses of these putative double crossover mutants indicated that two contained an intact PKS1 at the place of FUM1 in F. verticillioides (Fig. 5d) and the rest were revertants. The identity of the mutants was confirmed by Southern hybridization (Fig. 5e, f). Four probes were used in the Southern analysis. When the mixed $\mathrm{t} 1$ and $\mathrm{t} 2$ probes were used, mutant D4-1 gave two signals of 5.8 and $7.5 \mathrm{~kb}$ (Fig. 5e). The result confirms that D4-1 contains PKS1 in the place of FUM1 in F. verticillioides. As controls, the wild-type or a revertant (D4-5) did not give any signal, whereas the plasmid pUCH-LFUM1-PKS1-RFUM1 produced the expected 2.9 and $9.0 \mathrm{~kb}$ signals (Fig. 5e). When the mixed f3 and f1 probes were used, mutant D4-1 gave no signal (Fig. 5f). The wild-type or a revertant (D4-5) gave the expected 2.2 and $4.8 \mathrm{~kb}$ signals, whereas the plasmid pUCH-LFUM1PKS1-RFUM1 gave no signal (Fig. 5f). Together, the results show that D4-1 is a F. verticillioides mutant in which entire FUM1 gene has been replaced by $P K S 1$ gene.

The metabolites produced in the FUM1-replaced mutant as well as in the revertants of $F$. verticillioides were analyzed by HPLC-ELSD and LC-MS. The revertants produced a level and pattern of fumonisins similar to that of the wild-type (data not shown). However, the FUMI-replaced mutant did not produce any fumonisin or new analogs under the experimental conditions (data not shown). Extensive LC-MS search of the extracts from this mutant did not reveal any fumonisin-like or T-toxin-like metabolite.

\section{Discussion}

The genetic manipulation of PKS domains has proven to be a powerful way to elucidating the biosynthetic mechanism of bacterial polyketides $[12,25]$. However, this strategy is not directly applicable to fungal modular PKSs, because only a single set of domains is present in the fungal enzymes. In this study, we exploited this strategy by using two fungal PKSs that share similar domain architecture but synthesize different carbon chains. This approach has been used to study the biosynthesis of fungal aromatic polyketides in the recent years. For example, Watanabe and Ebizuka [30] constructed a chimeric PKS composed of domains from wA gene in A. nidulans [18] and pks1 gene in Colletotrichum langenarium [26]. These two genes code for PKSs synthesizing a heptaketide and a pentaketide, respectively. However, the chimeric PKS synthesized a novel hexaketide, showing the catalytic flexibility of the fungal PKS domains [30]. The genetic manipulation of domains, however, has not been conducted in the studies of fungal reduced polyketides due to the difficulties in the analysis of the acyclic, non-aromatic metabolites, as well as the challenge in genetically manipulating the filamentous fungi. We recently developed a genetic system for $F$. verticillioides that enables a specific manipulation of the domains of FUM1 [34]. In this study, we tested if a heterologous PKS and its KS domain could function in a chimeric FUM1 in this fungus.

FUM1 is the only PKS gene found in the 15-gene cluster (FUM) responsible for the biosynthesis of fumonisins in F. verticillioides $[20]$. The deduced protein (Fumlp) was pre- 
Figure 5 Screening and confirmation of the whole PKS replaced mutants. a Homologous recombination between FUMI on chromosome of $F$. verticillioides and the sequences on plasmid pUCH-LFUM1-PKS1RFUM1. The asterisk and filled circle indicate the position of the start and stop codon of the PKS genes, respectively. The positions of probes for Southern hybridizations are indicated by small bars. Abbreviations: Ap: ApaI; B: BamHI; $\operatorname{Hyg} B$, hygromycin $B$ resistant gene. $\mathbf{b}$ and $\mathbf{c}$ Gene disruption mutants resulted from a single crossover at the upstream homologous region (b) or at the downstream homologous region $(\mathbf{c})$. $\mathbf{d}$ The whole PKS replaced mutant resulted from a second crossover at the homologous regions. The expected sizes of hybridizing signals are indicated with arrows. $\mathbf{e}$ and $\mathbf{f}$ Confirmation of mutants by Southern hybridization using probes $\mathrm{t} 1+\mathrm{t} 2$ (e) or probes $\mathrm{f} 3+\mathrm{fl}(\mathbf{f})$. The DNA was digested with Bam HI and probed with DIG-labeled probes. Lane-1, size markers; lane-2, D4-1 (double crossover mutant); lane-3, D4-5 (revertant); and lane-4, plasmid (digested with ApaI)

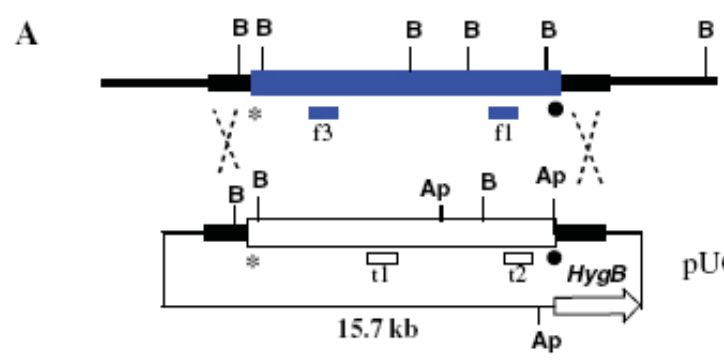

F. verticillioides chromosome

B

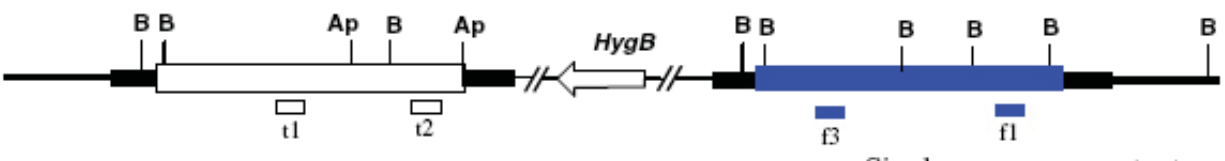

Single cross-over mutant
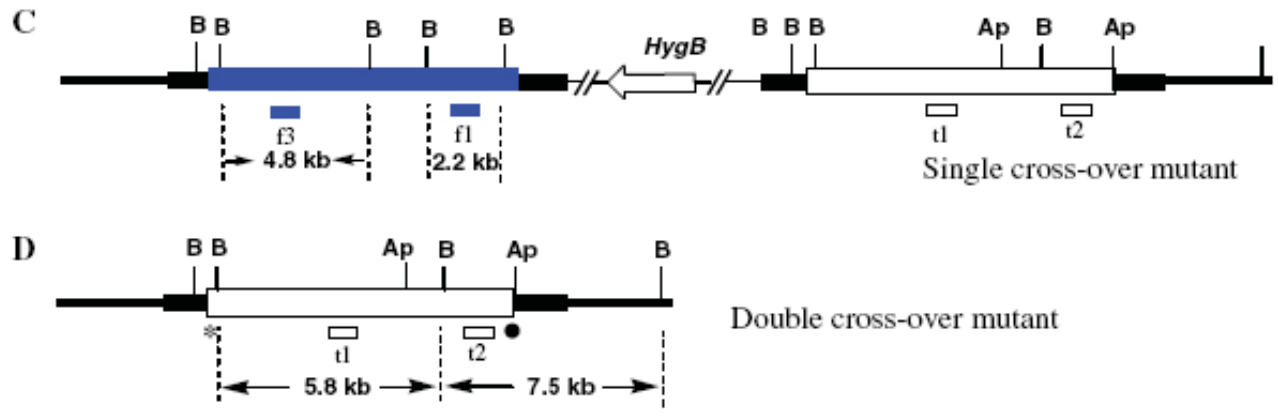

Double cross-over mutant
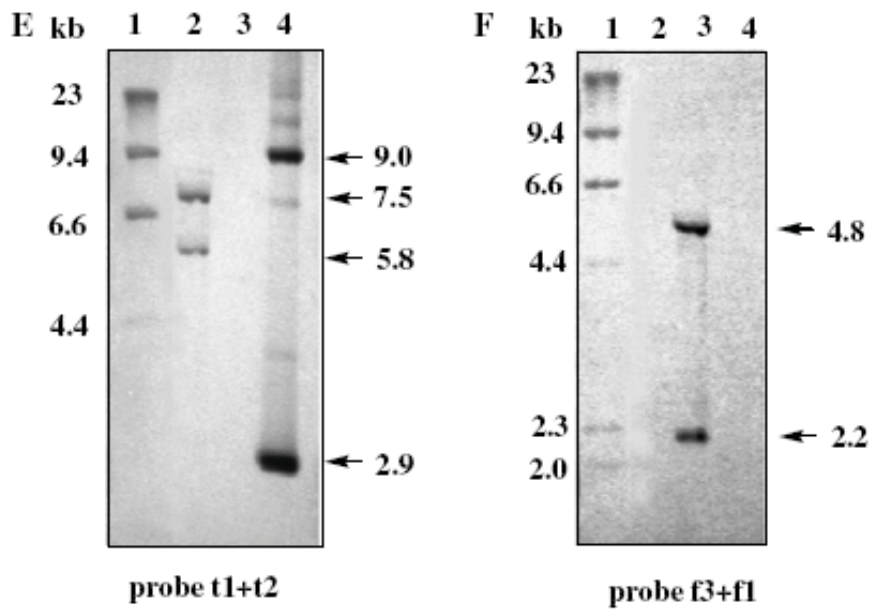

dicted to contain a single module, KS-AT-DH-MT-ER-KRACP [21]. Fum1p shares sequence similarity to many modular PKSs, with PKS1 for T-toxins showing the highest similarity (44.1\% similarity and $34.2 \%$ identity for the whole amino acid sequence). PKS1 is a gene involved in the biosynthesis of T-toxins in C. heterostrophus [32]. T-toxins are a family of mycotoxins with carbon chain lengths varying from $\mathrm{C}_{35}$ to $\mathrm{C}_{45}$, most of which have not been well characterized structurally, except the most abundant $(50 \%) \mathrm{C}_{41}$ analog (Fig. 1) [16]. When the KS domain of FUMI was replaced with the KS domain of $P K S 1$, the mutant strain produced fumonisins. The result has two interesting implications. First, the result shows that the KS domain of PKS1 can function in the context of Fum1p. Although many domains of bacterial PKSs have been success- fully replaced $[12,25]$, this is the first example of functional domain replacement in a PKS synthesizing a fungal reduced polyketide. The result suggests that, like the non-iterative bacterial PKSs, the domains of iterative fungal PKSs may be functionally manipulated, although these domains need to iteratively function in the biosynthesis of fungal polyketides. The level of products in the mutant was approximately $4 \%$ of that from the wild-type, suggesting that the hybrid enzyme may not be as active as the native form, probably due to sub-optimal interactions between the heterologous $\mathrm{KS}$ domain and the other domains in the chimeric enzyme or between the chimeric enzyme and the other biosynthetic enzymes in the fumonisin pathway. Second, the KS domain alone may not be sufficient to control the chain length of polyketide products. Among the domains of 
PKS, KS is the domain to catalyze the formation of new carbon-carbon bonds during the polyketide chain elongation. In bacterial type II PKS, the heterodimeric complex of ketosynthase and chain-length factor controls the chain length of aromatic polyketides $[14,28]$. Here, our data show that a switch of the KS domain did not lead to a new polyketide product. This suggests that the single module PKS for fungal reduced polyketides may have some yet-to-be-characterized factors/ features to control the structure of products. Under the experimental conditions used, no other metabolite, except fumonisins, was detected in the KS-replaced mutant. However, this does not exclude the possibility that the chimeric PKS may be producing other polyketides of different chain length, but these linear, fatty acid-like products may be metabolized by other pathways and, hence, not detected. This phenomenon has been observed in a previous study of other FUM mutants [5].

Since the fungal PKS appears flexible in the single domain replacement, we further tested if a replacement of the entire PKS could be tolerated. A mutant strain of $F$. verticillioides was generated, in which the entire FUM1 was replaced by PKSI. In order to keep a proper transcription and translation of the heterologous PKS gene in the new host, we only replaced the coding region of FUMI and did not make any changes to the upstream and downstream non-coding regions of FUM1. Indeed, RT-PCR results showed that the gene was expressed in the host (F. Yu and L. Du, unpublished data). Extensive HPLC-ELSD and LC-MS analyses showed that the mutant did not produce any fumonisin-like or T-toxin-like metabolite. Fungal PKSs for reduced polyketides do not contain a thioesterase/cyclase domain [11] and the release of the covalently attached polyketide intermediate from the PKS must rely on a mechanism different from the thioesterase/cyclase-dependent hydrolysis. In the biosynthesis of fumonisins, Fum8p, an $\alpha$-oxoamine synthase, has been proposed to carry out the polyketide chain release by a nucleophilic attack of the $\alpha$ carbon of alanine on the carbonyl carbon of polyketide acyl-S-PKS [5, 20]. This step not only releases the polyketide chain but also adds the two terminal carbons and the amino group of fumonisins $[5,29]$. Thus, the substrate specificity of Fum8p could be important for the production of a distinct product. We are currently undertaking both biochemical and genetic approaches to test this possibility.

\section{Acknowledgments}

We thank Drs. R. H. Proctor and R. D. Plattner at USDA, Peoria, IL, for providing Cos6B and standard fumonisins, Drs. O. C. Yoder and B. G. Turgeon at Cornell University, Ithaca, NY, for pF5P1, and Dr. David G. Gilchrist at University of California, Davis, CA, USA, for $F$. verticillioides wild-type strain A0149. Drs. Ron Cerny and Kurt Wulser at Nebraska Center for Mass Spectrometry, University of Nebraska-Lincoln, provided technical assistance in LC-ESMS analyses. This work was supported in part by an Oversea Young Scholar Cooperation Research Fund from the National Natural Science Foundation of China (No. 30428023), a Nebraska Redox Biology Center Pilot grant, and a NSF EPSCOR II grant.

\section{References}

1. Alexander NJ, Hohn TM, McCormick SP (1998) The TRI11 gene of Fusarium sporotrichioides encodes a cytochrome P-450 monooxygenase required for C-15 hydroxylation in trichothecene biosynthesis. Appl Environ Microbiol 64:221-225

2. Aparicio JF, Molnar I, Schwecke T, Konig A, Haydock SF, Khaw LE, Staunton J, Leadlay PF (1996) Organization of the biosynthetic gene cluster for rapamycin in Streptomyces hygroscopicus: analysis of the enzymatic domains in the modular polyketide synthase. Gene 169:9-16

3. Bedford DJ, Schweizer E, Hopwood DA, Khosla C (1995) Expression of a functional fungal polyketide synthase in the bacterium Streptomyces coelicolor A3(2). J Bacteriol 177:4544-4548 4. Blackwell BA, Edwards OE, Fruchier A, ApSimon JW, Miller JD (1996) NMR structural studies of fumonisin B1 and related compounds from Fusarium moniliforme. Adv Exp Med Biol 392:75-91

5. Bojja RS, Cerny RL, Proctor RH, Du L (2004) Determining the biosynthetic sequence in the early steps of the fumonisin pathway by use of three gene-disruption mutants of Fusarium verticillioides. J Agric Food Chem 52:2855-2860

6. Branham BE, Plattner RD (1993) Alanine is a precursor in the biosynthesis of fumonisin B1 by Fusarium moniliforme. Mycopath 124:99-104

7. Caldas ED, Sadilkova K, Ward BL, Jones AD, Winter CK, Gilchrist DG (1998) Biosynthetic studies of fumonisin B1 and AAL toxins. J Agric Food Chem 46:4734-4743

8. Cox RJ, Glod F, Hurley D, Lazarus CM, Nicholson TP, Rudd BA, Simpson TJ, Wilkinson B, Zhang Y (2004) Rapid cloning and expression of a fungal polyketide synthase gene involved in squalestatin biosynthesis. Chem Commun (Camb):2260-2261

9. Desjardins AE, Plattner RD, Proctor RH (1996) Genetic and biochemical aspects of fumonisin production. Adv Exp Med Biol 392:165-173

10. Ding Y, Bojja RS, Du L (2004) Fum3p, a 2-ketoglutarate-dependent dioxygenase required for C-5 hydroxylation of fumonisins in Fusarium verticillioides. Appl Environ Microbiol 70:1931-1934

11. Fujii I, Watanabe A, Sankawa U, Ebizuka Y (2001) Identification of Claisen cyclase domain in fungal polyketide synthase WA, a naphthopyrone synthase of Aspergillus nidulans. Chem Biol 8:189-197

12. Hopwood DA (1997) Genetic contributions to understanding polyketide synthases. Chem Rev 97:2465-2498

13. Kealey JT, Liu L, Santi DV, Betlach MC, Barr PJ (1998) Production of a polyketide natural product in nonpolyketide-producing prokaryotic and eukaryotic hosts. Proc Natl Acad Sci USA 95:505-509

14. Keatinge-Clay AT, Maltby DA, Medzihradszky KF, Khosla C, Stroud RM (2004) An antibiotic factory caught in action. Nat Struct Mol Biol 11:888-893

15. Kennedy J, Auclair K, Kendrew SG, Park C, Vederas JC, Hutchinson CR (1999) Modulation of polyketide synthase activity by accessory proteins during lovastatin biosynthesis. Science 284:1368-1372

16. Kono Y, Daly JM (1979) Characterization of the host-specific pathotoxin produced by Helminthosporium maydis race $\mathrm{T}$ affecting corn with Texas male sterile cytoplasm. Bioorg Chem 8:391-397

17. Marasas WF, Riley RT, Hendricks KA, Stevens VL, Sadler TW, Gelineauvan Waes J, Missmer SA, Cabrera J, Torres O, Gelderblom WC, Allegood J, Martinez C, Maddox J, Miller JD, Starr L, Sullards MC, Roman AV, Voss KA, Wang E, Merrill AH Jr (2004) Fumonisins disrupt sphingolipid metabolism, folate transport, and neural tube development in embryo culture and in vivo: a potential risk factor for human neural tube defects among populations consuming fumonisin-contaminated maize. J Nutr 134:711-716

18. Mayorga ME, Timberlake WE (1992) The developmentally regulated Aspergillus nidulans Wa gene encodes a polypeptide homologous to polyketide and fatty-acid synthases. Mol Gen Genet 235:205-212 
19. Plattner RD, Shackelford DD (1992) Biosynthesis of labeled fumonisins in liquid cultures of Fusarium moniliforme. Mycopath 117:17-22

20. Proctor RH, Brown DW, Plattner RD, Desjardins AE (2003) Co-expression of 15 contiguous genes delineates a fumonisin biosynthetic gene cluster in Gibberella moniliformis. Fungal Genet Biol 38:237-249

21. Proctor RH, Desjardins AE, Plattner RD, Hohn TM (1999) A polyketide synthase gene required for biosynthesis of fumonisin mycotoxins in Gibberella fujikuroi mating population A. Fungal Genet Biol 27:100-112

22. Richardson MT, Pohl NL, Kealey JT, Khosla C (1999) Tolerance and specificity of recombinant 6-methylsalicyclic acid synthase. Metab Eng $1: 180-187$

23. Sambrook J, Fritsch EF, Maniatis T (1989) Molecular cloning: a laboratory manual. Cold Spring Harbor Laboratory Press, Cold Spring Harbor, New York

24. Seo JA, Proctor RH, Plattner RD (2001) Characterization of four clustered and coregulated genes associated with fumonisin biosynthesis in Fusarium verticillioides. Fungal Genet Biol 34:155-165 25. Staunton J, Weissman KJ (2001) Polyketide biosynthesis: a millennium review. Nat Prod Rep 18:380-416

26. Takano Y, Kubo Y, Shimizu K, Mise K, Okuno T, Furusawa I (1995) Structural-analysis of Pks1, a polyketide synthase gene involved in melanin biosynthesis in Colletotrichum

27. Tang L, Yoon YJ, Choi CY, Hutchinson CR (1998) Characterization of the enzymatic domains in the modular polyketide synthase involved in rifamycin B biosynthesis by Amycolatopsis mediterranei. Gene 216:255265

28. Tang Y, Tsai SC, Khosla C (2003) Polyketide chain length control by chain length factor. J Am Chem Soc 125:12708-12709

29. Wang Q, Wang J, Yu F, Zhu X, Zaleta-Rivera K, Du L (2006) Mycotoxin fumonisins: health impacts and biosynthetic mechanism. Prog Nat Sci 16:7-15

30. Watanabe A, Ebizuka Y (2002) A novel hexaketide naphthalene synthesized by a chimeric polyketide synthase composed of fungal pentaketide and heptaketide synthases. Tetrahedron Lett 43:843-846

31. Watanabe A, Ebizuka Y (2004) Unprecedented mechanism of chain length determination in fungal aromatic polyketide synthases. Chem Biol 11:1101-1106

32. Yang G, Rose MS, Turgeon BG, Yoder OC (1996) A polyketide synthase is required for fungal virulence and production of the polyketide T-toxin. Plant Cell 8:2139-2150

33. Yi H, Bojja RS, Fu J, Du L (2005) Direct evidence for the function of FUM13 in 3-ketoreduction of mycotoxin fumonisins in Fusarium verticillioides. J Agric Food Chem 53:5456-5460

34. Yu F, Zhu X, Du L (2005) Developing a genetic system for functional manipulations of $F U M 1$, a polyketide synthase gene for the biosynthesis of fumonisins in Fusarium verticillioides. FEMS Microbiol Lett 248:257-264 